\title{
How can I help shielded trainees return to work?
}

\section{Trainees who have been shielding from covid-19 may now be able to return to work. Abi Rimmer asks experts about how they can be supported}

Abi Rimmer

\section{"Returning to work can be a psychological boost"}

GP Kamal Sidhu says, "Shielding trainees have been in a very difficult position. As well as worries around the health risks from covid-19, many may feel guilty for not being physically present during the pandemic. They're also likely to be worried about the impact shielding will have on their training.

"There'll also be apprehension around new policies and protocols that were implemented at their workplace while they were shielding. They may also have anxiety around how stringently all staff are following these safety measures.

"Returning to work, however, can be a good psychological boost after a long period of isolation, so it's vital to give these trainees the right support.

"The trainee's formal occupational health assessment will be important, although there will need to be room for flexibility and negotiation. A fresh formal risk assessment should also be considered, and it may still be safer for your trainee to continue to work remotely. If they do return to the workplace then a brief reinduction and shadowing, as well as a refresh on using tools such as Microsoft Teams, would be time well spent.

"Remember that the trainee may initially feel more confident starting with remote consulting in a covid-19-secure part of the workplace.

"The importance of regular contact after each clinical session and debriefs must not be underestimated. This isn't just an opportunity for the trainee to ask any burning questions but can be vital for informal support and any housekeeping.

"We mustn't forget that this group will need exceptional support in these exceptional times and any undue risk must be avoided."

\section{"Flexibility is key"}

Lucy Hanington, medicolegal consultant at Medical Protection Society, says, "Covid-19 has led to rapid and unprecedented changes in the practice of medicine. Shielding trainees will likely be returning to an environment that is very different to the one they left. New technologies, referral pathways, and policies may be in place. Targeted induction programmes will help these trainees feel equipped with the knowledge and skills to practise safely.

"A focus on health and wellbeing will also be crucial. Shielding trainees may have felt guilt or frustration about their inability to be in the workplace, and many will have ongoing health concerns. Teams may wish to consider how to provide colleagues with a warm welcome.

"Signposting appropriate sources of support will be important, and trainees will need to have access to regular risk assessments. The wider circumstances of each person will need consideration-financial worries, caring responsibilities, or bereavements may be additional sources of stress.

"Recent annual reviews of competence progression may have highlighted the impact shielding has had on training, leading to concern. Educational supervisors can help alleviate this concern by discussing training needs. Planning for how these can be met in uncertain times-including during further potential periods of shielding - may help to reduce anxiety and minimise the impact of covid-19 on career progression. Remote learning may also help to boost a CV while ticking off curriculum objectives.

"Trainees and their colleagues will need to be flexible and adapt to circumstances as they change- - a focus on individual needs is key."

\section{"Be empathetic and creative"}

Radha Sundaram, consultant in intensive care at the Royal Alexandra Hospital, Paisley, says, "Most shielding trainees, although physically removed from the workplace, have kept in touch with their peers and departments.

"Encourage them to seek an occupational health appointment so that an individual risk assessment can be carried out, along with getting tailored advice on risk mitigation at work. It's the employer's responsibility to ensure that these risk mitigation measures are put in place and, wherever possible, reasonable adjustments are made to support a return to work.

"Welcome them for an informal chat with their supervisor, either face-to-face or virtual, ahead of their first day at work. Confirm that they are fit tested for the personal protective equipment currently available at the hospital.

"Arrange shadowing shifts and a phased return. Recognise that along with their health vulnerabilities, these doctors will also have apprehensions around skill retention and team integration. Make sure that they have a mentor who they could meet for informal chats to discuss their experience. It also helps to prepare the team that they are joining so that insensitive remarks about a prolonged break are not made.

"Ensure that the team adheres to social distancing rules. Having posters about face mask wearing and 
social distancing are good visual reminders for the others. Recognise that they cannot just resume routine duties-be perceptive and responsive to their views and needs.

"Our teams are not complete without these doctors-be empathetic and creative about maximising their training opportunities while protecting them."

\section{"You are their supervisor and not their occupational health physician"}

Jonathan Corne, postgraduate dean, East Midlands, says, “Although shielding came to an end on 1 August, many healthcare workers will not be able to return to their normal work environment because of clinical vulnerability.

"One way you can help is to recognise the disruption, stress, and uncertainty that shielding causes. Some trainees will have returned to work following a period of shielding and be concerned about the need to catch up on competencies.

"Working from home or in another non-clinical environment does not mean not working at all, and it's important that clinical training opportunities are maximised. These may include virtual

consultations, radiology or pathology reporting, or interpretation of physiological tests. Many of these virtual skills will be particularly appropriate as these become a standard part of clinical practice.

"Employers should provide the equipment necessary to undertake clinical work remotely and, as a supervisor, you should ensure that adequate clinical supervision can be provided. This may be through Microsoft Teams, three way virtual consultations, or a thorough debrief following a virtual clinic or reporting session.

"The development of non-clinical, generic skills should also be facilitated. These are easily achievable outside the clinical environment and may include leadership projects such as quality improvement, and educational projects such as the development of virtual learning modules.

"Educational supervision is the responsibility of both the trainee and trainer but it's much more difficult when trainees are based outside the clinical environment. It is important to be proactive in contacting your trainees and doing so on a regular basis. Use these meetings to discuss their clinical and non-clinical work, remain updated on any plans for their return, provide pastoral support and advise them on any other support available.

"At a point when they are about to return, work with them to plan how they can catch up on competencies, for example by dual site working, changes to their programme, or out of programme training. Remember, though, that you are their supervisor and not their occupational health physician, and any decision as to the safety of return to work is outside your remit.

"Finally, in England, there is a huge bank of resources, including support-to-training programmes, that are available for all shielding trainees returning to work. ${ }^{12}$ There is also a careers service for those contemplating a change in specialty or leaving medicine altogether. You may not be an expert when it comes to many of the problems your trainees will face, but there will be someone in your deanery who is and will be happy to give you advice."

\section{"Treat them as individuals"}

Hilary Tedd, consultant in respiratory medicine and Health Education England North East (HEENE) supported return to training (SuppoRTT) lead, Linda Waddilove, consultant anaesthetist and HEENE SuppoRTT lead, and Tricia Campbell, specialty registrar in orthopaedics and HEENE leadership fellow, say, “As SuppoRTT leads, we typically support trainees who have had periods away from clinical work for parental leave, research, or sick leave. The shielding necessitated by covid-19 has created a new group of potential returners, with no established pathway or precedent.

"We recognised that although this group will likely face challenges typical of most returners, they may also experience unique concerns and practical difficulties determined by each person's circumstances.

"In order to identify what the trainees felt to be their biggest challenges, we organised a remote meeting with shielding trainees across all specialties. Our aims were to improve their experience both during shielding and on returning to clinical practice.

"Recurrent themes included working in isolation, anxiety about their own health, confidence problems, guilt about being off, and concerns about future periods of shielding. There was a huge sense of uncertainty regarding impact on training and what they 'should' be doing during shielding, compounded by lack of available answers from conventional sources.

"Subsequently, we set up a peer-to-peer network, aiming to ease the sense of isolation and held a regional webinar that covered donning and doffing, updates on advanced life support algorithms, and the impact of low confidence when returning to work. An open forum session enabled further discussion around specific anxieties and challenges faced by individual trainees.

"As they return, it's important to recognise and acknowledge each shielding trainee, including their level of risk, their training requirements, and their particular concerns. Key interventions to ensure a safe and supported return may include liaison with occupational health, regular check-ins with supervisors, bespoke clinical timetables, continued flexibility to access teaching remotely, IT resources to enable remote working, and a proactive plan for any future period of shielding."

Health Education England. SuppoRTT: digital and online resources. www.hee.nhs.uk/ourwork/doctors-training/supporting-doctors-returning-training-after-time-out/supportt-digital-onlineresources.

2 Health Education England. Coronavirus (covid-19) information for trainees. www.hee.nhs.uk/coronavirus-information-trainees. 